\title{
SENSIBILIDAD Y RESISTENCIA DE GÉRMENES INTRA Y EXTRA HOSPITALARIOS EN EL HOSPITAL INFANTIL UNIVERSITARIO DE LA CRUZ ROJA DE MANIZALES, 2007
}

\author{
IOVAN A. GALVIS MONROY \\ LEONARDO BARRERA ARIZA ${ }^{2}$
}

Manizales, 2010-04-29 (Rev. 2010-07-26)

\section{RESUMEN}

Siempre que se plantea la resistencia antibiótica como un problema ambiental, no se debe centrar solamente en el ámbito hospitalario, pues también se debe tener en cuenta que el uso que se dé a los antibióticos se puede extender al medio ambiente donde se vive o, simplemente, originarse desde el medio ambiente externo y migrar al ámbito hospitalario. Por esta razón, se deben tener en cuenta las relaciones interespecíficas que caracterizan nuestro planeta, así como las relaciones humanas con los microorganismos, reconociendo el papel esencial de estos en el mantenimiento de la actividad médica y farmacéutica, que ha dado lugar a la sensibilidad y a la resistencia de diferentes patógenos a través del uso de antibióticos. La resistencia de los microorganismos es muy desesperanzadora. Desde el punto de vista de la clínica y experiencia práctica, cada vez se observa un mayor aumento de la resistencia antibiótica, que ocasiona dificultad en la implementación de un tratamiento adecuado. Pocas estrategias encaminadas en el control de este problema realmente están funcionando en la actualidad.

\section{PALABRAS CLAVE:}

Antibióticos, resistencia y sensibilidad bacteriana, infecciones, antibiograma, hombre, sociedad.

\section{SENSITIVITY AND RESISTANCE OF INTRA AND EXTRA HOSPITAL GERMS IN THE MANIZALES RED CROSS CHILDREN'S UNIVERSITY HOSPITAL, 2007}

\begin{abstract}
Whenever antibiotic resistance is posed as an environmental problem, it shouldn't be centered only in the hospital setting. The use given to antibiotics must also be considered because it might be extended to the surrounding environment or, simply it might be originated in external surroundings and migrate to the hospital setting. For this reason, the inter-specific relationships which characterize our planet must be taken into consideration, as well as the human relationships with microorganisms, recognizing their essential role in the maintenance of the medical and pharmaceutical activity, which has generated the sensibility and resistance of different pathogens through the use of antibiotics. The resistance of microorganisms is hopeless. From the clinic and practical experience, more and more a greater increase in the antibiotic resistance is observed which causes difficulty in the implementation of an adequate treatment. Few strategies aimed to the control of this problem are really working presently.
\end{abstract}


KEY WORDS: antibiotics, bacterial resistance and sensitivity, infections, antibiogram, man, society.

\section{INTRODUCCIÓN}

En el marco de las relaciones interespecíficas que caracterizan nuestro planeta, las relaciones de los humanos con los microorganismos han sido abordadas desde múltiples perspectivas. Reconociendo el papel esencial de los microorganismos en el mantenimiento del fenómeno "vida planetaria", en este estudio optamos por caracterizar esta relación desde las modificaciones que la actividad farmacéutica y médica ha generado en la sensibilidad y resistencia de los diferentes gérmenes patógenos mediante el uso de antibióticos.

El manejo antibiótico adecuado depende de muchos factores, tales como el conocimiento adecuado de la flora bacteriana más frecuente en el ambiente donde vivimos y el conocimiento de la sensibilidad y la resistencia de estos gérmenes a los distintos antibióticos. La clara identificación de estos aspectos nos da la posibilidad de poner en práctica planes de manejo empírico de mayor efectividad que eviten el aumento de la inefectividad terapéutica.

En nuestro medio, el tratamiento de los procesos infecciosos se basa en el diagnóstico clínico, de tal manera que la elección del antibiótico que se vaya a emplear, se realiza de forma empírica o fundamentada en estudios norteamericanos. De igual manera, la prescripción de antibióticos se realiza en muchas ocasiones antes de conocer los resultados de los estudios microbiológicos, y es frecuente que para su elaboración no se tenga en cuenta el perfil epidemiológico de la localidad, ni los agentes etiológicos más prevalentes en el medio, ni el perfil de sensibilidad de los mismos.

El objetivo de este trabajo consistió en identificar los gérmenes etiológicos del ámbito intra y extra hospitalario, de las muestras correspondientes a los procesos infecciosos de mayor frecuencia del Hospital Infantil Universitario de la Cruz Roja "Rafael Henao Toro", así como su perfil de sensibilidad y resistencia antibiótica, mediante el análisis de la información del laboratorio de microbiología de la institución. Se tuvo en cuenta que la información referida puede contribuir, a futuro, a la construcción de guías de manejo específicas para ciertas patologías en las cuales los microorganismos identificados como los más frecuentes se encuentran implicados.

\section{MATERIALES Y MÉTODOS}

El estudio se realizó en el Hospital Infantil Universitario de la Cruz Roja "Rafael Henao Toro" de la ciudad de Manizales, el cual maneja una población comprendida en el grupo etáreo entre un mes y 18 años de edad.

La investigación consistió en la realización de un estudio descriptivo retrospectivo de los urocultivos, coprocultivos, frotis faríngeos, hemocultivos y cultivos de líquido cefalorraquídeo procesados en el laboratorio de microbiología del Hospital, entre diciembre de 2006 y diciembre del 2007, los cuales provenían de los servicios de Consulta Externa, Urgencias, Unidad de Quemados, Unidad 
Quirúrgica, Unidad de Cuidados Intensivos, Pensión, Hematología, Oncología, $\begin{array}{llll}\text { Pediatría } & y & \text { Unidad }\end{array}$

La información se obtuvo de las bases de datos físicas del laboratorio de microbiología. Se incluyeron los registros que tenían asignado el código del examen, la fecha, el nombre y el género del paciente, el tipo de muestra, el servicio al que pertenece, el agente aislado y los antibióticos que mostraron sensibilidad y resistencia. Estos datos, en total 454 registros, se tabularon en Epilnfo versión 0.6. Las variables contempladas fueron fecha, género, tipo de muestra, servicio, agente etiológico y antibióticos. Los antibióticos tuvieron como categorías: sensible, resistente y sin dato. Esta última variable se utilizó para mostrar que el antibiótico no había sido usado. En el estudio no se incluyeron exámenes cuyo resultado no presentaba antibiograma, tampoco los registros cuyos reportes presentaban errores en su interpretación, eran ilegibles o estaban incompletos. Los servicios considerados como intrahospitalarios fueron Hematología, Oncología, Pediatría, Pensión, Unidad de Quemados, Unidad Quirúrgica, Unidad de Cuidados Intensivos y Unidad Renal. Los servicios considerados como extra hospitalarios fueron Consulta Externa y Urgencias.

\section{RESULTADOS}

En el estudio se analizaron 454 registros, de los cuales el 51,3\% correspondió a pacientes de género masculino y el $48,6 \%$ restante al femenino. En la Figura 1 puede apreciarse la distribución de los casos por género y servicio hospitalario.

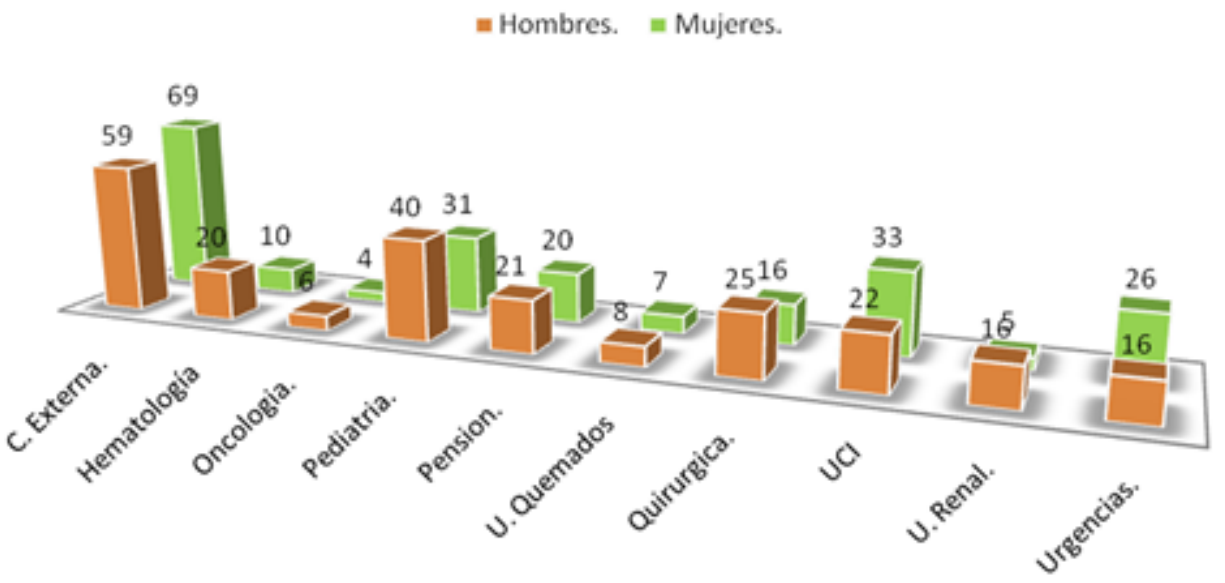

Figura 1. Distribución de pacientes por género y servicio hospitalario en el Hospital Infantil Universitario de la Cruz Roja "Rafael Henao Toro", entre diciembre de 2006 y diciembre de 2007.

Los servicios hospitalarios involucrados fueron diez: Consulta Externa (128 muestras), Hematología (30 muestras), Oncología (10 muestras), Pediatría (71 muestras), Pensión (41 muestras), Unidad de Quemados (15 muestras), Unidad Quirúrgica (41 muestras), Unidad de Cuidados Intensivos (55 muestras), Unidad Renal (21 muestras) y Urgencias (42 muestras). Su distribución porcentual puede observarse en la Figura 2. 


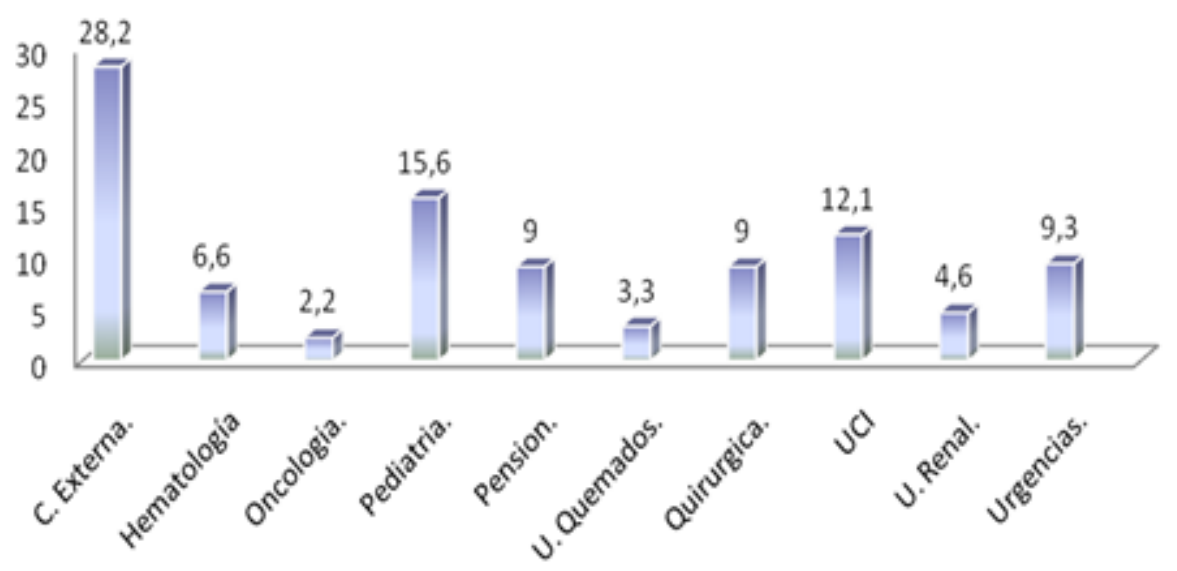

Figura 2. Porcentaje de muestras por servicio en el Hospital Infantil Universitario de la Cruz Roja "Rafael Henao Toro", entre diciembre de 2006 y diciembre de 2007.

Los tipos de muestra obtenidos fueron cinco: coprológico (43 muestras), frotis faríngeo (1 muestra), hemocultivo (143 muestras), líquido cefalorraquídeo (7 muestras) y orina (260 muestras). Su distribución porcentual puede revisarse en la Figura 3.

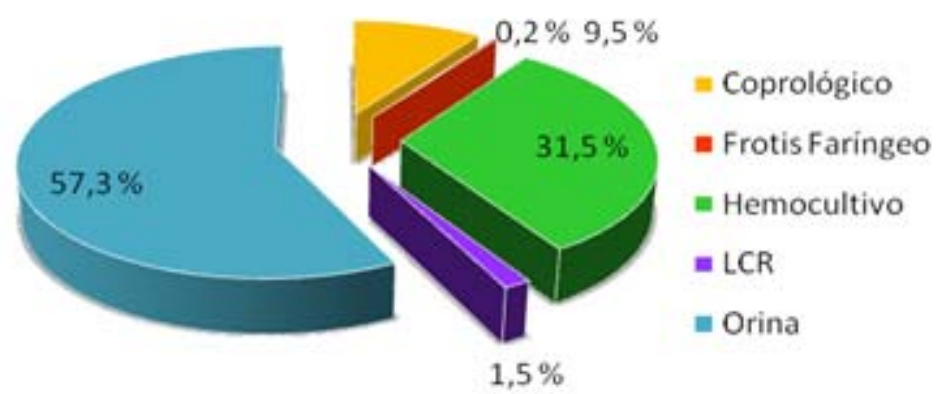

Figura 3. Porcentaje según el tipo de muestra en el Hospital Infantil Universitario de la Cruz Roja "Rafael Henao Toro", entre diciembre de 2006 y diciembre de 2007.

Tabla 1. Frecuencia y porcentaje respectivo del tipo de muestra en el ámbito extra e intra hospitalario en el Hospital Infantil Universitario de la Cruz Roja "Rafael Henao Toro" entre diciembre de 2006 y diciembre de 2007.

\begin{tabular}{|c|c|c|c|c|c|c|}
\hline Ambito & Coprologico & $\begin{array}{c}\text { Frotis } \\
\text { Faringeo }\end{array}$ & Hemocultivo & LCR & Uroanálisis & Total \\
\hline $\begin{array}{c}\text { Extra } \\
\text { hospitalario }\end{array}$ & $6(3,5 \%)$ & $1(0,6 \%)$ & $7(4,1 \%)$ & $1(0,6 \%)$ & $155(91,2 \%)$ & 170 \\
\hline $\begin{array}{c}\text { Intrahospitala } \\
\text { rio }\end{array}$ & $37(13 \%)$ & $0(0 \%)$ & $136(47,9 \%)$ & $\begin{array}{c}6 \\
(2,1 \%)\end{array}$ & $105(37 \%)$ & $\mathbf{2 8 4}$ \\
\hline Total & 43 & 1 & 143 & 7 & 260 & 454 \\
\hline
\end{tabular}


De 454 muestras totales, $170(37,4 \%)$ corresponden al ámbito extra hospitalario y $284(62,6 \%)$ al ámbito intra hospitalario.

Del total de 76 agentes etiológicos aislados, se destacan los cinco primeros. Estos fueron: Escherichia coli, que representó el 36,6\% del total de agentes aislados; Staphylococcus DNAsa negativo con un 8,1\%; Proteus mirabilis con un 6,8\%; Klebsella pneumoniae con 4,8\% y Staphylococcus aureus con un 4,2\%.

Al realizar la discriminación por género, se observa predominio del género femenino en el ámbito extra hospitalario y predominio del género masculino en el ámbito intra hospitalario (Figura 4).

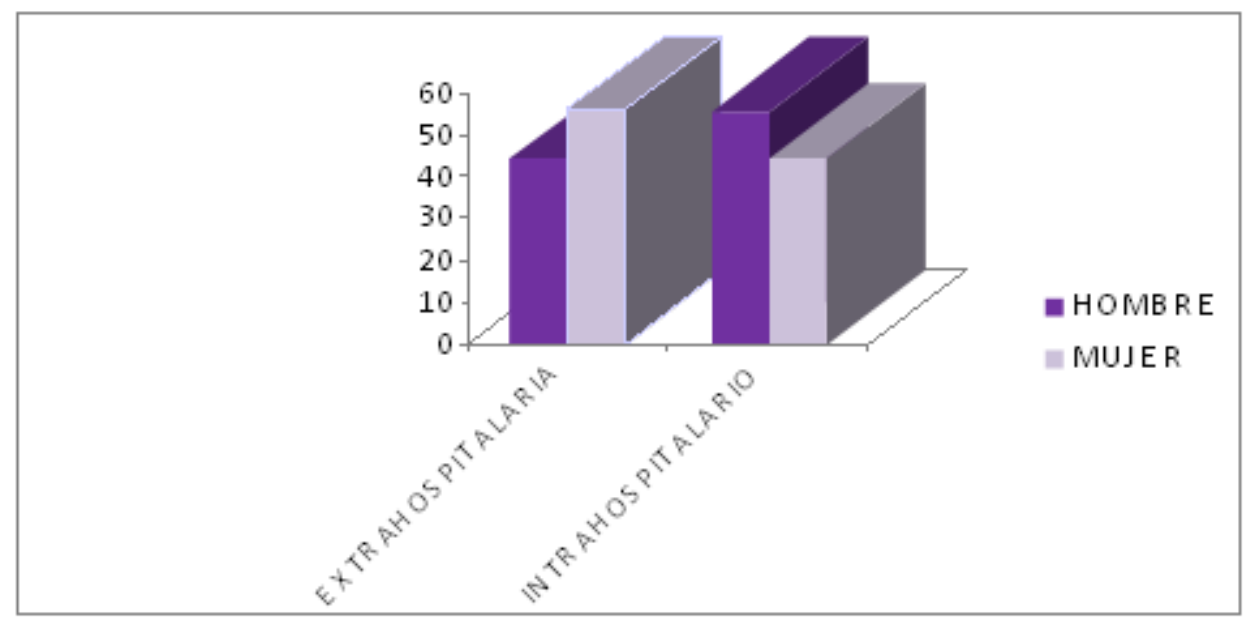

Figura 4. Frecuencia de géneros en el ámbito extra e intra hospitalario en el Hospital Infantil Universitario de la Cruz Roja "Rafael Henao Toro", entre diciembre de 2006 y diciembre de 2007.

Tabla 2. Agentes etiológicos más frecuentes en el ámbito extra hospitalario en el Hospital Infantil Universitario de la Cruz Roja "Rafael Henao Toro", entre diciembre de 2006 y diciembre de 2007.

\begin{tabular}{|lc|}
\multicolumn{1}{|c}{ Agente etiológico. } & Frecuencia. \\
\hline Escherichia coli. & $99(58,2 \%)$ \\
\hline Proteus mirabilis. & $22(12,9 \%)$ \\
\hline Proteus vulgaris. & $6(3,5 \%)$ \\
\hline Klebsella pneumoniae. & $6(3,5 \%)$ \\
\hline Pseudomona aeruginosa. & $4(2,3 \%)$ \\
\hline Staphylococcus DNAsa negativo. & $4(2,3 \%)$ \\
\hline
\end{tabular}

Tabla 3. Agentes Etiológicos más frecuentes en el ámbito intrahospitalario en el Hospital Infantil Universitario de la Cruz Roja "Rafael Henao Toro", entre diciembre de 2006 y diciembre de 2007. 


\begin{tabular}{|lc|}
\hline \multicolumn{1}{|c|}{ Agente etiológico } & Frecuencia. \\
\hline Escherichia coli. & $67(23,6 \%)$ \\
\hline Staphylococcus DNAsa negativo. & $33(11,6 \%)$ \\
\hline Klebsella pneumoniae. & $18(6,3 \%)$ \\
\hline Staphylococcus aureus. & $16(5,6 \%)$ \\
\hline Staphylococcus DNAsa positivo. & $14(4,9 \%)$ \\
\hline \hline Proteus mirabilis. & $10(3.5 \%)$ \\
\hline
\end{tabular}

Perfil de sensibilidad y resistencia de los principales agentes etiológicos extra e intra hospitalarios:

\section{Agentes etiológicos extra hospitalarios}

En la Gráfica 1 se evidencia que Escherichia coli es más sensible a la amikacina (80\%), nitrofurantoina (79\%) y ciprofloxacina (60\%). Presenta mayor resistencia antibiótica a trimetropin sulfa (46\%), ampicilina (29\%) y ampicilina sulbactam (22\%).

Grafica 1. Sensibilidad y resistencia de Escherichia coli en el ambito extrahospitalario.
口SENSIBLE

QRESISTENTE

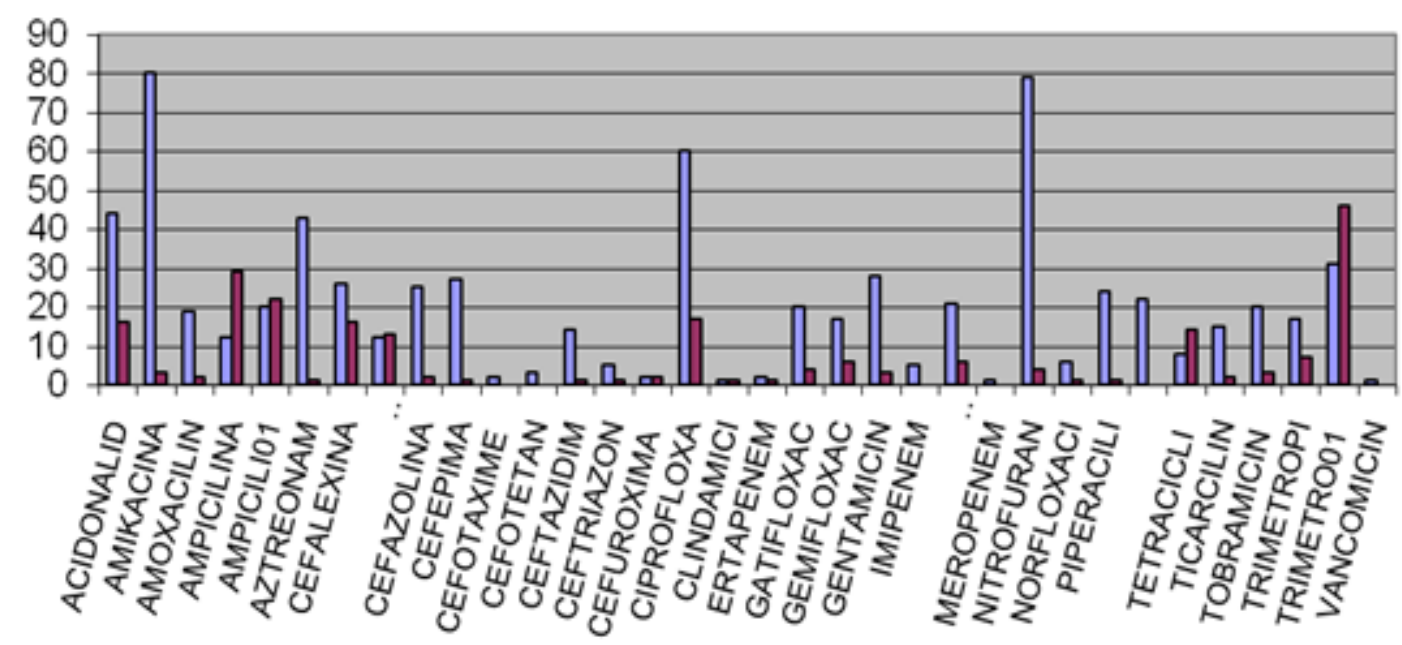

En la Gráfica 2 se evidencia que Proteus mirabilis es más sensible a la ciprofloxacina (86,3\%), amikacina (63,6\%) y ampicilina sulbactam $(54,4 \%)$. Presenta mayor resistencia antibiótica a nitrofurantoina (63,3\%), ácido nalidíxico y amikacina (13,6\% cada uno). 
Grafica 2. Sensibilidad y resistencia de Protens mirabilis en el ambito extrahospitalario.

QSENSIBLE

QRESISTENTE
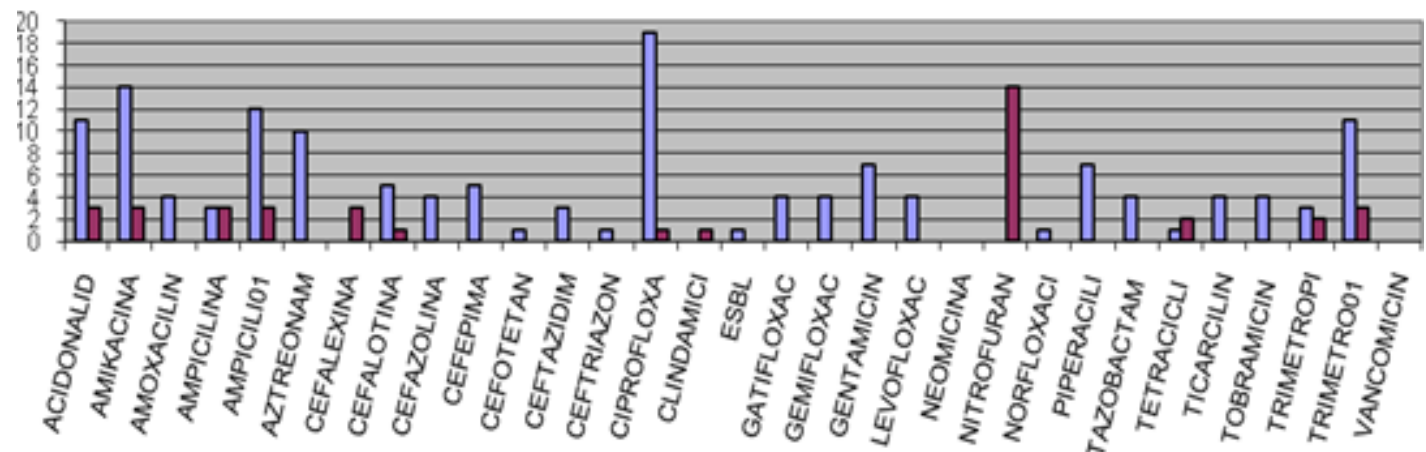

En la Gráfica 3 se evidencia que Proteus vulgaris es más sensible a la amikacina (100\%), aztreonam, ciprofloxacina y gentamicina (66,6\% cada uno). Presenta mayor resistencia antibiótica al ácido nalidíxico, cefalexina y nitrofurantoina (33,3\% cada uno).

Grafica 3. Sensibilidad y resistencia de Proteus vulgarisen el ambito extrahospitalario.

DSENSIBLE

aRESISTENTE

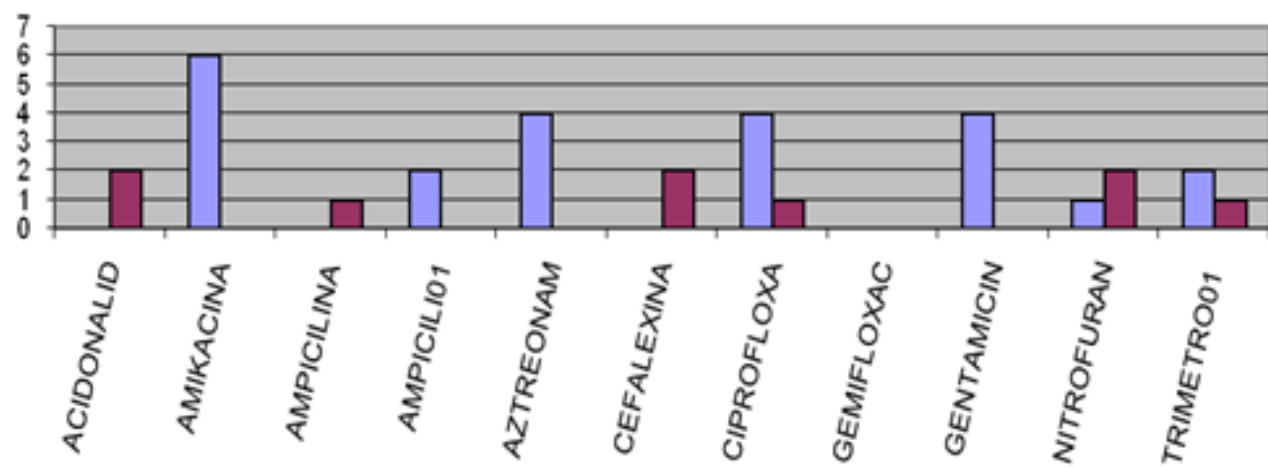

En la Gráfica 4 se evidencia que Klebsella pneumoniae es más sensible a la amikacina y nitrofurantoina $(66,6 \%$ cada uno) y ácido nalidíxico (33,3\%). Presenta mayor resistencia antibiótica a la ampicilina sulbactam $(66,6 \%)$, ácido nalidíxico (33,3\%) y ampicilina (16,6\%). 

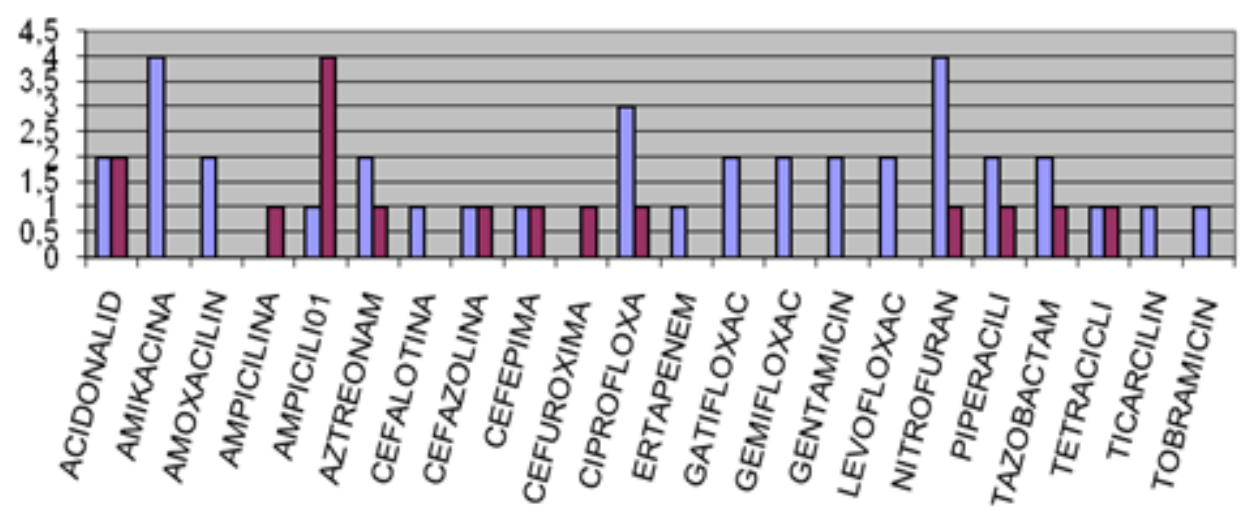

En la Gráfica 5 se evidencia que Pseudomona aeruginosa es más sensible en un $100 \%$ de los casos a piperacilina, amikacina y ceftazidima. No se evidenció resistencia para algún agente antimicrobiano.

Grafica 5. Sensibilidad y resistencia de Pseudomona aeruginosa en el ambito extrahospitalario.

\section{口SENSIBLE} QRESISTENTE

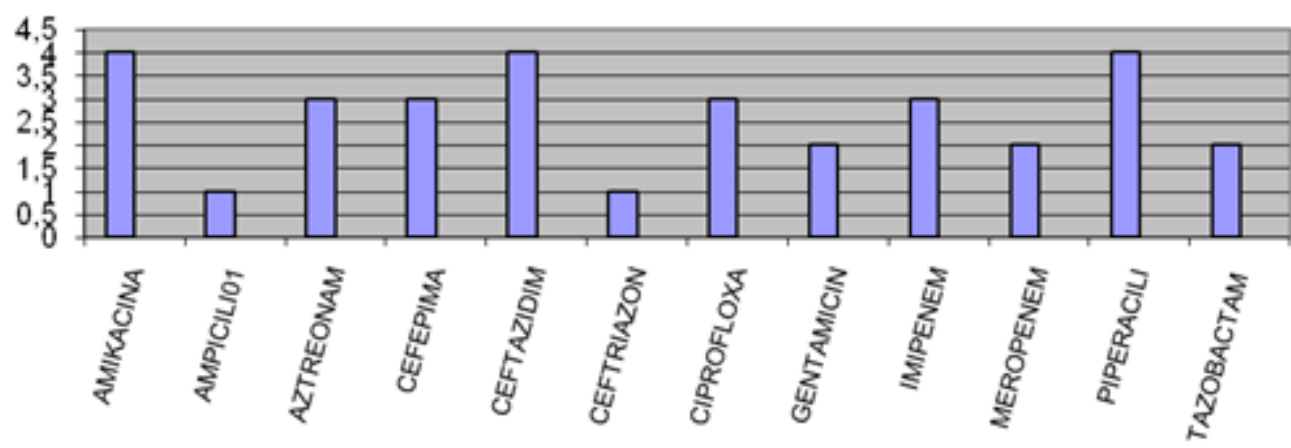

En la Gráfica 6 se evidencia que Klebsella pneumoniae es más sensible a la ciprofloxacina (75\%), eritromicina y trimetropin sulfa (50\% cada uno). Presenta mayor resistencia antibiótica a la clindamicina y oxacilina ( $50 \%$ cada uno) y al ácido nalidíxico (25\%). 


\section{Grafica 6. Sensibilidad y resistencia Staplylococcus DNAsa negativo en el ambito extrahospitalario.

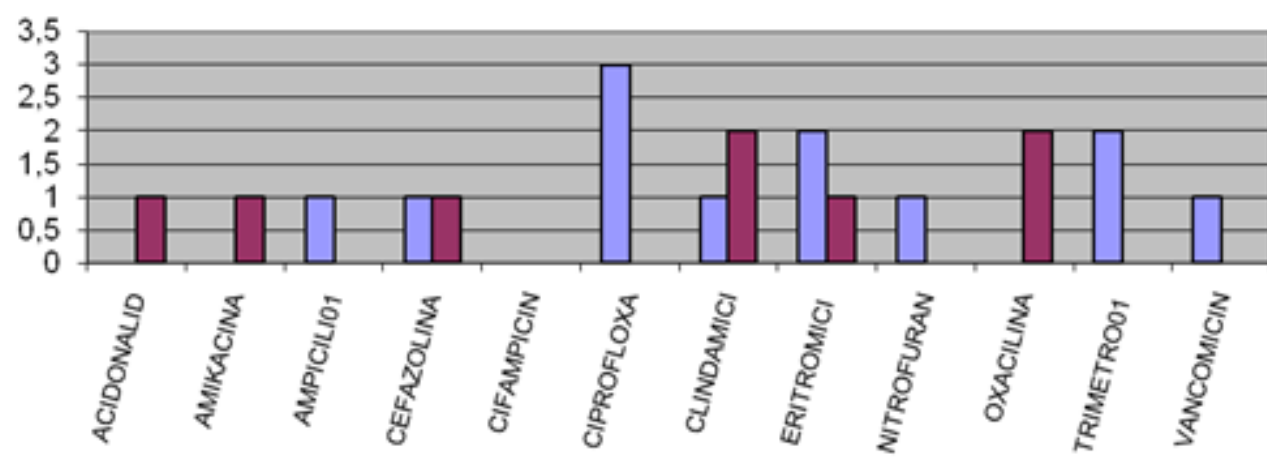

\section{Agentes etiológicos intra hospitalarios}

En la Gráfica 7 se evidencia que Escherichia coli es más sensible a la amikacina (73\%), ciprofloxacina (64\%) y nitrofurantoina (53,7\%). Presenta mayor resistencia antibiótica a trimetropin sulfa (32,8\%), ampicilina y ampicilina sulbactam $(20,8 \%$ cada uno).

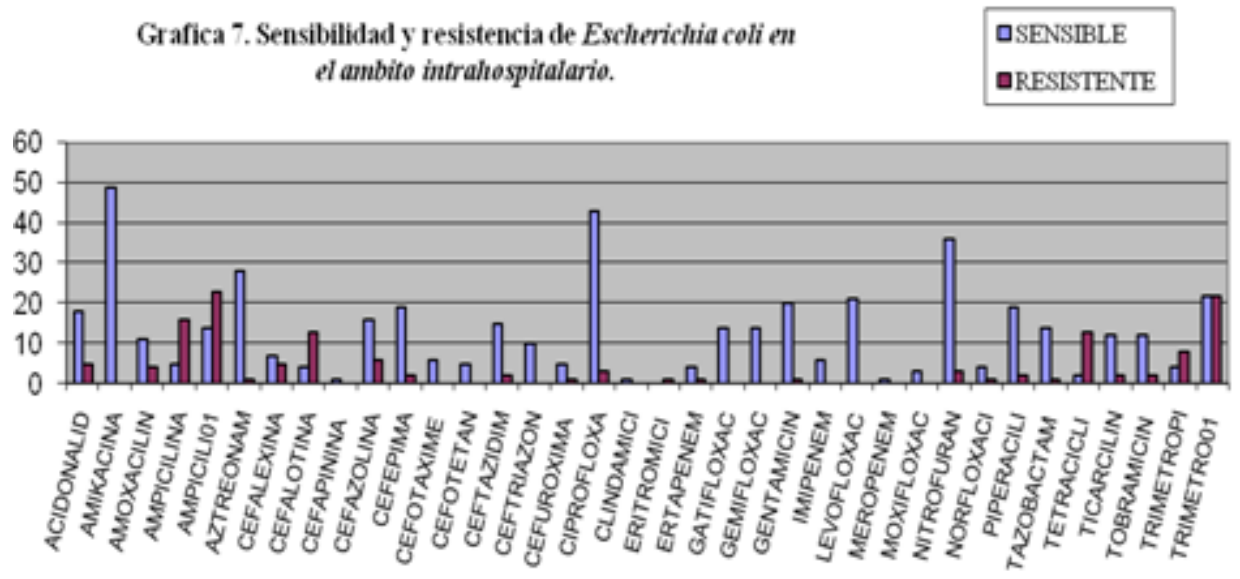

En la Gráfica 8 se evidencia que Satphylococcus DNAsa negativo es más sensible a la amikacina (75\%), ciprofloxacina $(63,6 \%)$ y clindamicina $(54,5 \%)$. Presenta mayor resistencia antibiótica a la cefazolina $(42,4 \%)$, eritromicina y oxacilina (39,3\% cada uno). 


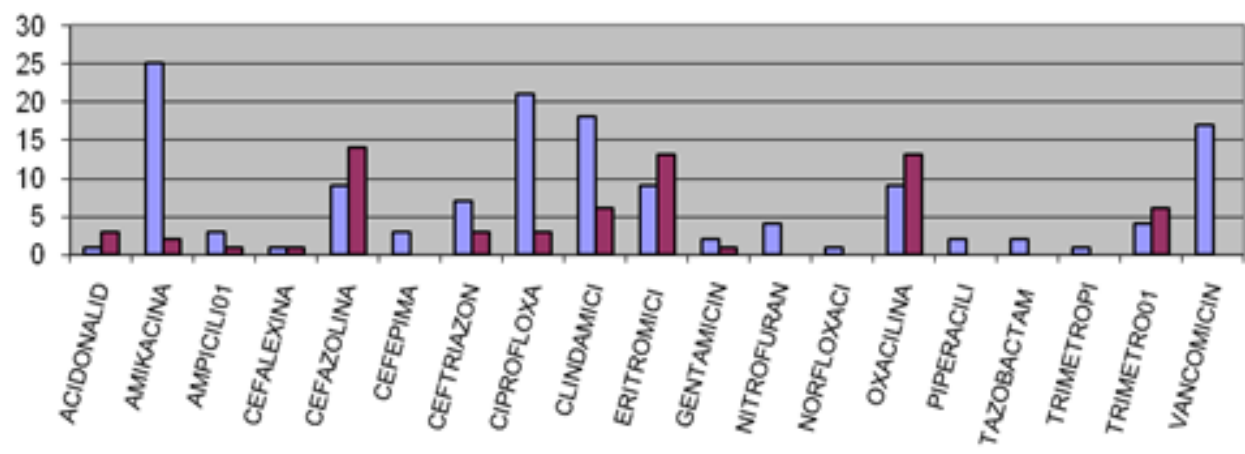

En la Gráfica 9 se evidencia que Klebsiella pneumoniae es más sensible a la ciprofloxacina $(72,2 \%)$, amikacina y gentamicina (44,4\% cada uno). Presenta mayor resistencia antibiótica a la ampicilina sulbactam $(27,7 \%)$ y cefalotina $(22,2 \%)$.

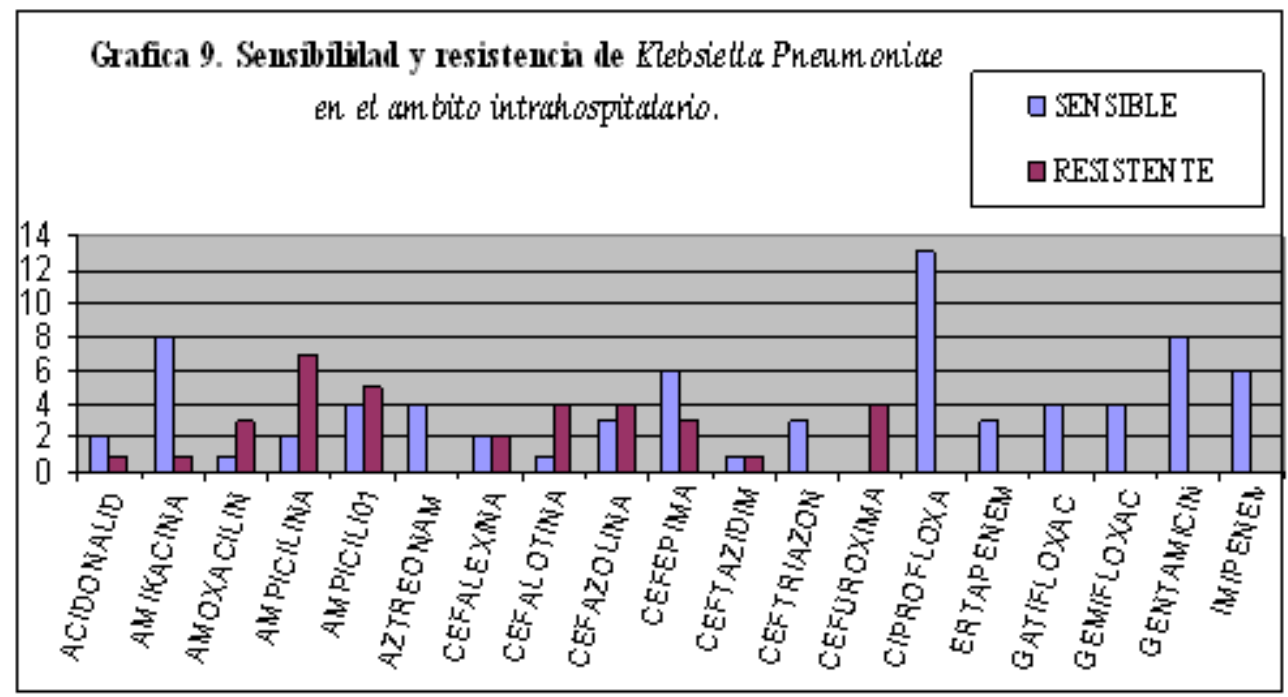

En la Gráfica 10 se evidencia que Satphylococcus aureus es más sensible a la gentamicina (100\%), ciprofloxacina y vancomicina (93,7\% cada uno). Presenta mayor resistencia antibiótica a la oxacilina (50\%), eritromicina $(68,7 \%)$ y clindamicina $(12,5 \%)$. 


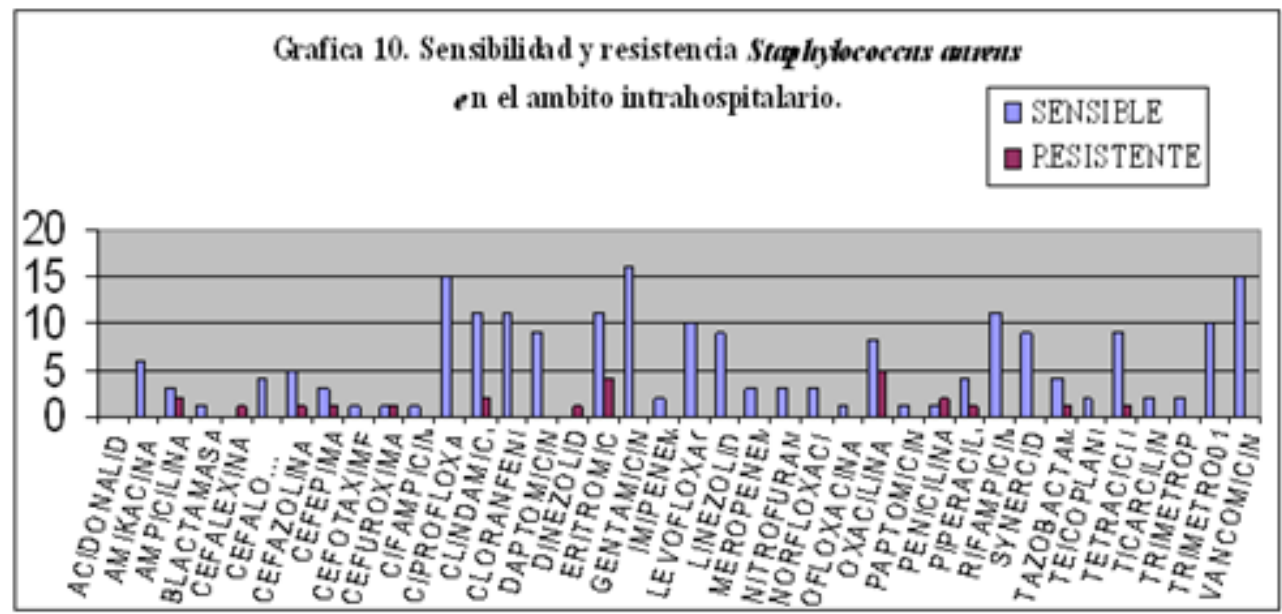

En la Gráfica 11 se evidencia que Satphylococcus DNAsa positivo es más sensible a la ciprofloxacina y clindamicina (85,7\% cada uno) y amikacina (57\%). Presenta mayor resistencia antibiótica a la cefazolina y oxacilina $(42,8 \%)$ y a la eritromicina $(28,5 \%)$.

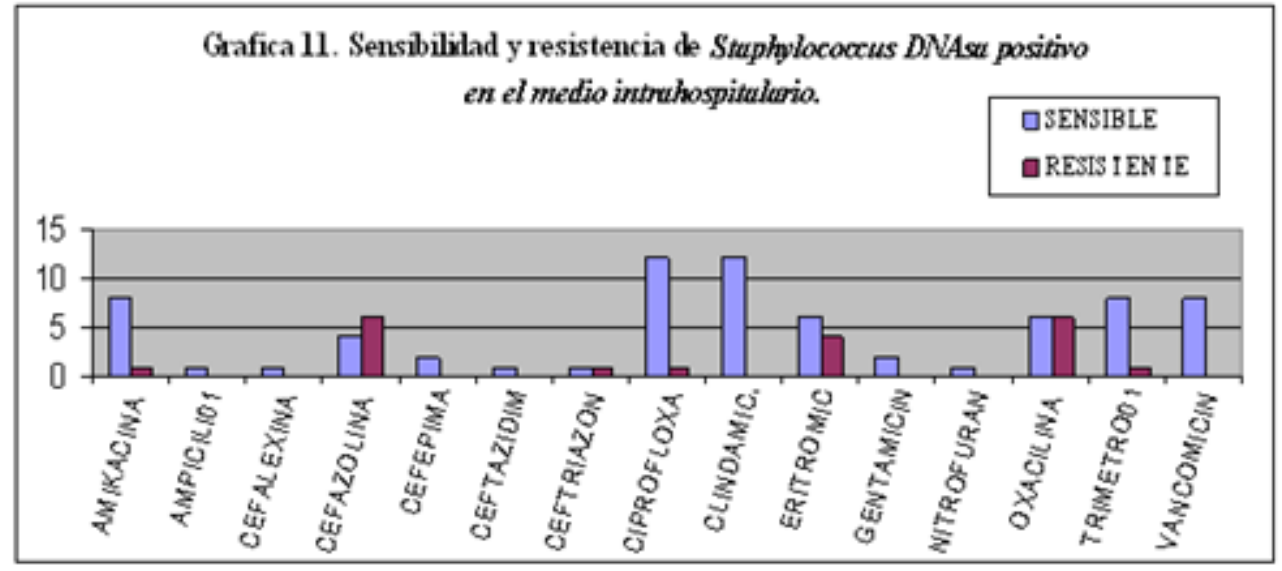

En la Gráfica 12 se evidencia que Proteus mirabilis es más sensible a la ciprofloxacina (90\%), amkacina, ampicilina sulbactam y trimetropin sulfa (80\% cada uno). Presenta mayor resistencia antibiótica a notrofurantoina (50\%), cefalexina (40\%) y tetraciclina (30\%).

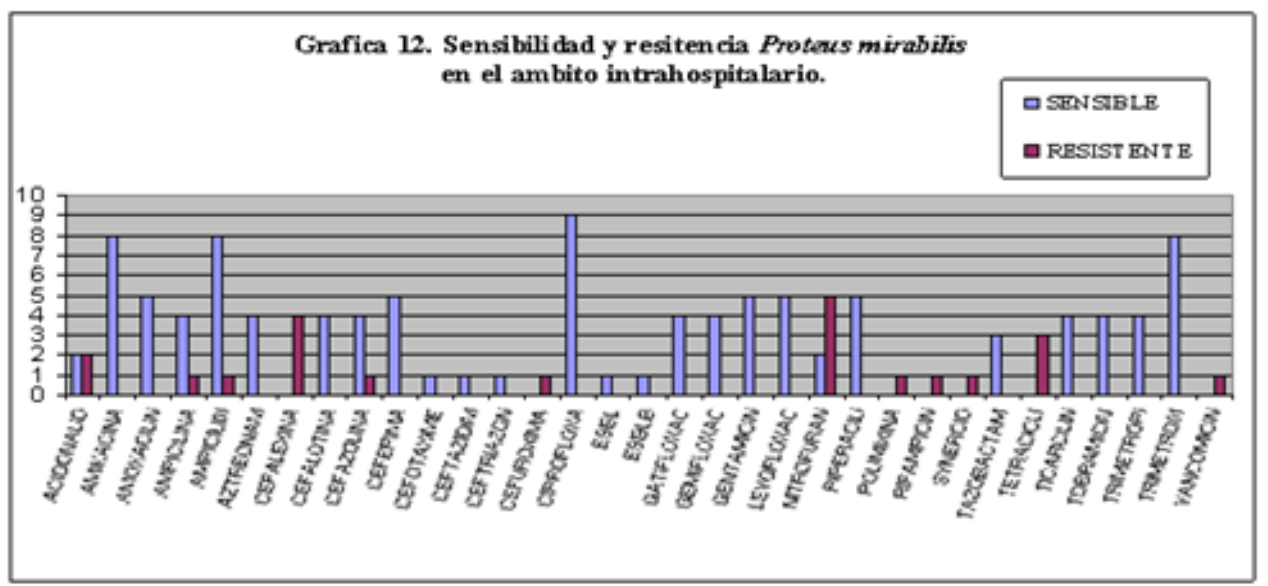




\section{DISCUSIÓN y CONCLUSIONES}

Al momento de plantearse la resistencia antibiótica como un problema ambiental, se debe no solo centrar en el ámbito local hospitalario, ya que también debe tenerse en cuenta que el uso que se haga de los antibióticos se puede extender al medio ambiente donde se vive o, simplemente, originarse desde el medio ambiente externo y migrar al ámbito hospitalario. Además, hay que considerar que los antibióticos son parte de nuestro arsenal terapéutico más empleado, en la batalla que emprendimos contra los agentes biológicos que impactan en la salud humana y animal. Gran parte de este arsenal es utilizado en el manejo de pacientes hospitalizados, quienes pueden recibir uno o más antibióticos, algunos de estos como parte de un tratamiento antibiótico inapropiado basado en el empirismo medico.

La perspectiva de la resistencia de los microorganismos a los agentes antibióticos es muy desesperanzadora. Desde el punto de vista de la práctica y experiencia clínica, cada vez se observan mayores índices de resistencia antibiótica que ocasionan dificultad en la implementación de un tratamiento terapéutico adecuado. Pocas estrategias encaminadas en el control del problema están realmente funcionando en la actualidad. Debemos controlar de una forma racional el uso de herramientas desarrolladas, que pretenden ofrecernos mejor calidad de vida, teniendo en cuenta que nuestro entorno ?la Naturaleza? seguirá su curso con o sin el hombre como habitante del planeta Tierra. La sociedad tiene que cambiar sus hábitos de consumo y la actitud frente al medio ambiente si deseamos continuar viviendo en el planeta.

En la revisión de la literatura científica se evidencia un incremento importante del arsenal terapéutico y, por lo tanto, del arsenal antibiótico en los últimos 50 años aproximadamente. De igual manera, se percibe un aumento en el número de microorganismos resistentes a los agentes antimicrobianos.

Un estudio demostró que las complicaciones de las peritonitis producidas por bacterias resistentes, tales como la formación de abscesos y la re-operación, se duplican cuando la terapia antibiótica no es la adecuada para este tipo de bacterias. Este hecho es sólo uno de los múltiples casos de complicación de una patología secundaria a la resistencia antibiótica. La aparición de la resistencia antibiótica de las bacterias, además de ser un problema biológico, es sin lugar a dudas un problema médico, social, económico y ético, dado que las infecciones producidas por estas bacterias resistentes a los antibióticos conllevan mayor morbilidad y mortalidad. Un concepto importante para destacar es que incluso con la terapia antibiótica clínicamente adecuada, el antibiótico genera la selección de bacterias resistentes en la flora normal del individuo tratado y de su entorno. Es así como los antibióticos, mediante procesos como la farmacocinética y farmacodinamia, pueden ser aerosolizados y pasar al ambiente a través de la piel y las excretas, incluyendo sudor, orina y deposiciones, conservando la capacidad antibiótica y proyectando esta actividad al entorno temporal y espacial del paciente.

El desarrollo del proceso de la resistencia antibiótica de las bacterias es claramente explicado por una síntesis que reúne la teoría de la evolución de Darwin con el conocimiento de la plasticidad del material genético bacteriano.

El incremento de la resistencia bacteriana ante los agentes terapéuticos agrega mayor costo en el tratamiento de las infecciones producidas, y ocasiona cuadros 
clínicos más severos y más difíciles de tratar, debido a que los mismos elementos genéticos responsables de la resistencia pueden contener genes que aumenten la virulencia bacteriana.

Consideramos que el conocimiento previo y el uso racional del arsenal terapéutico de que disponemos, así como la identificación de la biota de nuestro ambiente en particular y de sus características intrínsecas y extrínsecas, nos permitirá adquirir herramientas valiosas que estarán a la disposición de la salud humana. En caso contrario, el conocimiento adquirido ?cuyo objetivo inicial apuntaba al bienestar social y personal? y empleado de forma errónea, se puede convertir en un elemento maléfico si se le da un uso inadecuado y poco racional.

Este trabajo de investigación informa sobre el comportamiento de las bacterias resistentes y sensibles en el medio extra e intra hospitalario en el Hospital Infantil "Rafael Henao Toro", centro de referencia en el departamento de Caldas. La información referida contribuirá en la construcción de guías de manejo específicas para ciertas patologías en las que los microorganismos identificados y los más frecuentes se encuentran implicados; del mismo modo, le permite al médico tratante la selección adecuada y racional del antibiótico que proveerá mejor respuesta terapéutica, reduciendo así los costos que implicaría el uso del antibiótico inadecuado y de los días de estancia hospitalaria. A la sociedad le permitirá conocer las patologías más frecuentes en un nicho particular y le posibilitará generar estrategias de promoción y prevención en los grupos poblacionales que deberían tener mayor intervención.

En el periodo de investigación se identificó que de la población consultante, el $51,3 \%$ eran hombres y el $48,6 \%$ mujeres, y que los hombres presentaron menor frecuencia de consulta extra hospitalaria, pero tenían mayor frecuencia de hospitalización. En el género femenino el comportamiento fue inverso. De las hospitalizaciones realizadas, los servicios que solicitaron el mayor porcentaje de exámenes son Pediatría y la Unidad de Cuidados Intensivos. En estos servicios se considera importante la utilización de la información reportada para la implementación de guías de manejo antibiótico que permitan disminuir la aparición de resistencia antibiótica.

La identificación de los agentes etiológicos más frecuentes implicados en las patologías a las que se enfrentan los médicos en el contexto extra e intra hospitalario, les permitirá enfocar un manejo oportuno y racional acerca de la impresión diagnóstica que se da en el primer contacto médico-paciente, generando un manejo terapéutico distante del empirismo clínico, basado en información objetiva y real del medio donde realiza su quehacer profesional.

Cuando se analizan los resultados sobre la sensibilidad y resistencia antibiótica de los agentes etiológicos del ámbito extra e intra hospitalario, se pretende reportar toda la información hallada con el fin de que sea el criterio medico, basado en la individualidad del paciente, en el entorno económico y social, el que determine la elección del esquema terapéutico adecuado.

Finalmente, hay que decir que existen ciertas diferencias en cuanto a lo expuesto en la literatura y lo que se encuentra en nuestro ambiente, y aunque no sean de gran magnitud, pueden ser parte importante de un buen manejo antibiótico. $Y$ más aún cuando en nuestro medio el manejo inicial de cualquier agente etiológico es empírico. 


\section{AGRADECIMIENTOS}

Al Hospital Infantil Universitario de la Cruz Roja "Rafael Henao Toro", y a la Dra. Luz Elena Sepúlveda Gallego, por su asesoría estadística y epidemiológica.

\section{BIBLIOGRAFÍA}

- Agüero, M.; Deluca, A.; Timmis, K. \& Cabello, F. (1984). "A plasmidencoded outer membrane protein, TraT, enhances resistance of Escherichia coli to phagocytosis". Infect Immun, 46, 740-746.

- Bronzwaer, Stef. (2003). European antimicrobial resistance surveillance as part of a Community strategy. University of Groningen . Netherlands.

- Cabello, F. (2004). "Antibiotics and aquaculture in Chile: Implications for human and animal health". Rev Méd Chile, 132, 1001-1006.

- Cantón Moreno, R. (2002). "Lectura interpretada del antibiograma: ¿ejercicio intelectual o necesidad clínica?". Enferm Infecc Microbiol Clin, 20(4), 176-86.

- Carranza, M. A.; Rodríguez, D. \& Díaz, J. (2003). "Etiología y resistencia bacteriana de las infecciones urinarias en pacientes hospitalizados en el Centro Médico Naval entre enero y diciembre del 2003". Rev Soc Per Med Inter, 16(3).

- Cornejo, M. (s.f.). "La Selección del Antibiótico". Revista Médica del C.I.E.M. [Hospital Nacional del Sur de Arequipa (HNSA) - Instituto Peruano de Seguridad Social. Universidad Católica de Santa María].

- Cosgrove, S.; Saye, K.; Eliopoulos, G. \& Carmeli, Y. (2000). "Health and economic outcomes of the emergence of third generation cephalosporin resistance in Enterobacter species". Arch Intern Med, 162, 185-190.

- Courvalin, P. \& Trieu-Cuot, P. (2001). "Minimizing potential resistance: The molecular view". Clin Infect Dis, 33(Suppl 3), S138-S146.

- Daza Pérez, R. M. (1998). "Resistencia bacteriana a antimicrobianos: su importancia en la toma de decisiones en la práctica diaria". Inf Ter Sist Nac Salud, 22, 57-67.

- Diestraa, K.; Coquebc, T. M.; Miróa, E. \& Oteod, J. (2008). "Caracterización y epidemiología molecular de betalactamasas de espectro extendido en Escherichia coli y Klebsiella pneumoniae en once hospitales españoles (2004)". Enferm Infecc Microbiol Clin 26(7), 404-10.

- Drobnic, L. (1997). "Principios generales del tratamiento antibiótico". En: Tratamiento Antimicrobiano. Madrid: Emisa. pp. 639-650.

- Foxman, B. (2003). "Epidemiology of urinary tract infections: Incidence, morbidity and economic costs". Dis Mon, 49(2), 53-70.

- García Rodríguez J. A. \& García Sánchez, E. (s.f.). "Resistencias bacterianas y antibioterapia". En: Eficacia in vivo Eficacia in vitro. MadridBarcelona: Doyma. pp. 39-50.

- Harrison, P. \& Lederberg, J. (1998). Antimicrobial Resistance: Issues and Options. Workshop Report. Washington, DC: National Academy Press.

- Holmberg, S. D.; Solomon, S. L. \& Blake, P. A. (1987). "Health and economic impacts of antimicrobial resistance". Rev Infect Dis, 9, 10651078.

- Jaramillo, E. L. (1996). "Vigilancia epidemiológica de infecciones intrahospitalarias. Hospital de Caldas 1989-1993". Colombia Médica, 27, 21-25. 
- Leal Castro, A. L. MD. Msc. (s.f.). Impacto de la resistencia bacteriana en la salud pública. Universidad Nacional de Colombia Grupo para el Control de la Resistencia Bacteriana en Bogotá.

- Levy, S. (2001a). "Antibiotic resistance: Consequences of inaction". Clin Infect Dis, 33 (Suppl 3): S124-S129. Publishing (2001b). The antibiotic paradox. Cambridge, MA: Perseus Lindsay, N. (2003). "Best Pharmacological practice: Urinary tract infections". Expert Opin Pharmacother, 4(5), 693-704.

- Mazón, A.; Gil Setas, A. \& López Andrés, A. (2002). "Etiología y sensibilidad antibiótica de las infecciones extrahospitalarias más frecuentes, excepto las del tracto respiratorio inferior". ANALES. Sis San Navarra, 25(3): 273-280.

- Mesa Española de Normalización de la Sensibilidad y Resistencia a los Antimicrobianos (MENSURA). Recomendaciones del grupo MENSURA para la selección de antimicrobianos en el estudio de la sensibilidad.

- Mosdell, D.; Morris, D.; Voltura, A.; Pitcher, D.; Twiest, M.; Milne R. et al. (1991). "Antibiotic treatment for surgical peritonitis". Ann Surg, 214, 543549.

- Quintero, G. (s.f.). "Reciclaje de los antibióticos". Revista Colombiana de Cirugía. SCC., FACS, FRCS.

- Robinson, A.; Marcon, M.; Mortensen, J. E.; McCarter, Y. S.; LaRocco, M.; Peterson, L. R. et al. (1999). "Controversies affecting the future of clinical microbiology". J Clin Microbiol, 37, 883-889.

- Rubin, R.; Harrington, C.; Poon, A.; Dietrich, K.; Greebe, J. \& Moiduddin, A. (1999). "The economic impact of Staphyloccocus aureus infection in New York City hospitals". Emerg Infect Dis, 5, 9-17.

- Shnayerson, M. \& Plotkin, M. (2002). The killers within. The deadly rise of drug-resistant bacteria. Boston, MA: Back Bay Books. Little, Brown and Co.

- Warren, J. W.; Abrutyn, E; Hebel, J. R.; Johnson, J. R.; Schaeffer, A. J. \& Stamm, W. E. (1999). "Guidelines for Antimicrobial Treatment of Uncomplicated Acute Bacterial Cystitis and Acute Pyelonephritis in Women Clinical Infectious Diseases, 29. pp. 745-58.

1.Estudiante de medicina de la Universidad de Caldas.

2.Estudiante de medicina de la Universidad de Caldas. 